\title{
Finite Element Analysis of a PTO Shaft Used in an Agricultural Tractor
}

\section{H. Kursat Celik ${ }^{1 *}$, Recep CINAR ${ }^{2}$, Gokhan KUNT¹ , Allan E.W. RENNIE ${ }^{3}$, Mehmet UCAR ${ }^{4}$ and Ibrahim AKINCI ${ }^{1}$}

${ }^{1}$ Department of Agricultural Machinery \& Technology Engineering, Faculty of Agriculture, Akdeniz University, Turkey

${ }^{2}$ Design and Engineering Bureau, Yenasoft Inc., Turkey

${ }^{3}$ Lancaster Product Development Unit, Engineering Department, Lancaster University, UK

${ }^{4}$ Department of Automotive Engineering, Faculty of Technology, Kocaeli University, Turkey

\section{Research Article}

Volume 2 Issue 3

Received Date: May 05, 2018

Published Date: May 15, 2018

DOI: $10.23880 /$ eoij-16000147

*Corresponding author: H. Kursat CELIK, Department of Agricultural Machinery \& Technology Engineering, Faculty of Agriculture, Akdeniz University, 07070, Antalya, Turkey, Tel: +90 24231065 70; Email: hkcelik@akdeniz.edu.tr

\section{Abstract}

This study describes a finite element method (FEM) based deformation simulation procedure for a power take off (PTO) shaft in an agricultural tractor. The agricultural tractor is a mobile power source in agricultural fields. The Agricultural tractor transmits power to the working implement through several systems independently. Most especially, rotary elements used in agricultural machinery take the required power and movement from the tractor take off (PTO) shaft. During this operation, the PTO shaft experiences a high dynamic loading condition such as excessive instant (impact) loading. This may cause an undesired failure case for the PTO shaft. In order to prevent such undesired failures, loading condition and stress distribution on the component should be described properly; however, an accurate description of the structural stress distribution on the shaft becomes an important problem. In this content, a case study was carried out on a failed PTO shaft, as described in this paper. The aim of this case study is to exhibit the stress distribution on the PTO shaft through finite element analysis under a torsional loading case which may be considered as the main cause of the failure. Visual outputs from the simulation results revealed a better understanding of the failure zone on the shaft. The maximum equivalent stress magnitude obtained from the simulation was 632.08 [MPa] (which was lower than the fracture point) on the shaft under maximum PTO torque, however, it was concluded that the main reason for the failure was excessive shock torsional loading. This work contributes to further research into usage of numerical method based deformation simulation studies for the transmission elements used in agricultural tractors/machinery.

Keywords: Design of Agricultural Machinery; Finite Element Analysis; Stress Analysis; Engineering Simulation; PTO Shaft Failure; Shock Loading 


\section{Ergonomics International Journal}

\section{Introduction}

Nowadays, food/agricultural production issues are becoming more important as the world population rapidly increases and future predictions indicate that providing enough food for the population is becoming a serious problem. It is clear that enough food can be produced from larger agricultural fields and this can be sustainable with implementation of larger agricultural mechanisation systems in the production. Hence, this increase in the world population has necessitated an increase in the number of agricultural tractors and machinery in many countries in the world. In parallel, the tractor population in Turkey has reached a high of 1.27 million and the agricultural machinery used in production (animal and plant production) has been declared as 10.5 million in 2016 [1]. Although the amount of machinery is important (one of the indicators of the level of agricultural mechanisation in a country is the population of agricultural tractors and machinery), use of efficient mechanisation tools in the field has become a key parameter in efficient food production. In this context, optimum design and manufacturing issues for agricultural machinery/equipment in the industry should be considered; however, experiencing the failures on the machinery/equipment used in the agricultural operations is an unavoidable matter. Every year people are seriously injured, or even killed, in accidents involving failures of machine elements used in agricultural machinery systems such as power take-off (PTO) shafts [2].

Analysing mechanical failures occurring in different mechanical systems has interested engineers since the first mechanical systems designed in the history. In failure analysis, engineers mainly use three methods which are analytical, numerical and experimental. In solving complex engineering problems, analytical and experimental methods may uncover problematic aspects which can be solved by numerical methods only. Therefore, nowadays, it would be correct to say that the combination of hi-tech powerful computers and numerical methods integrated with skilful engineering software has becomeindispensable in mechanical design and failure investigation studies. Most especially, finite element method (FEM) based engineering analysis, which is one of the most popular numerical approaches used in the engineering research area, provides a good understanding for complex design/engineering problems. Therefore, FEM based engineering simulations have been widely used in engineering fields of study. One of the most important engineering analyses carried out through FEA is structural stress analysis which is a complete and comprehensive study of stress distribution in a mechanical component [3]. Use of numerical methods based engineering analysis techniques in design studies of agricultural machinery systems is also vital for efficient agricultural production.

Agricultural tractors play an important role in agricultural production as a mobile power source. The Agricultural tractor transmits power to the working implement through several systems independently. Most especially, rotary elements used in the agricultural machinery take the required power and movement from the tractor take-off (PTO) shaft. In an agricultural tractor, required rotational power is transmitted from the engine through a gear train to the PTO shaft. During this operation, the PTO shaft experiences a high torsional loading which may cause failure of the PTO shaft. The PTO shaft may also experience different types of failures because of unpredictable dynamic working conditions such as shock loading, design, material and/or operator faults; in any case, it is necessary to describe the main cause of failure of the PTO shaft in order to prevent undesired failure cases during operation.

In this paper, a stress analysis case study for a failed PTO drive shaft used in an agricultural tractor was carried out. A finite element method (FEM) based deformation simulation procedure for the failed PTO shaft is discussed in detail within this study. Visual outputs from the simulation results are presented and this helps to provide a better understanding of the failure zone on the shaft. Suggestions for preventing similar failures on the PTO shaft have also been discussed.

\section{Materials and Methods}

\section{Failure of the PTO Shaft}

In this study, a failed PTO shaft used in an agricultural tractor (TUMOSAN 6170 4WD) has been considered. The shaft failure occurred during field operation of a chopper machine used for pellet production processes. On first inspection, fast brittle fracture signs have been observed on the fracture surfaces of the shaft. Initial observations of the shaft and shaft bearing led us to consider a shock loading case (shock torsion) or material fault; however, it was not possible to predict the exact loading type and magnitude at the failure up on initial visual investigation. In order to clarify the loading effect and stress distribution at the point of failure on the PTO shaft, a FEA was utilised with due consideration of critical material properties such as fracture point of the PTO shaft. Some technical specifications of the tractor and visuals taken from the failed PTO shaft are shown in Figure 1 and Figure 2 respectively. 


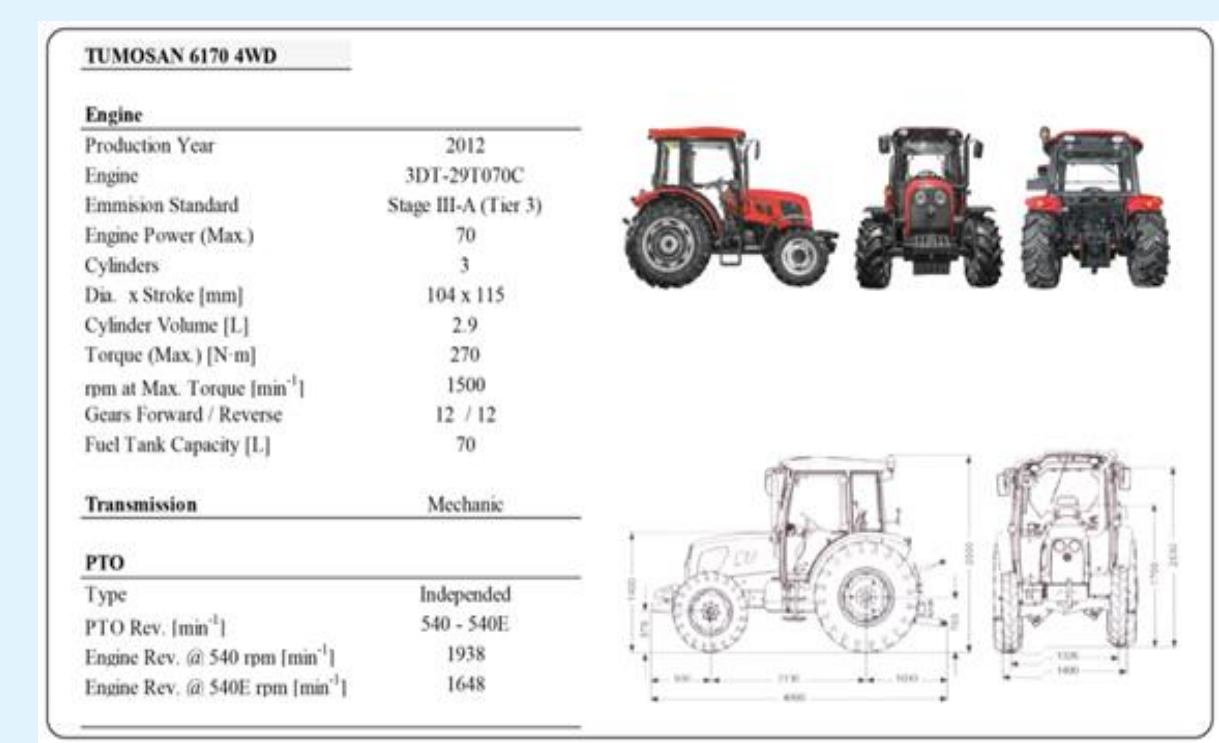

Figure 1: Tractor Technical Specifications.

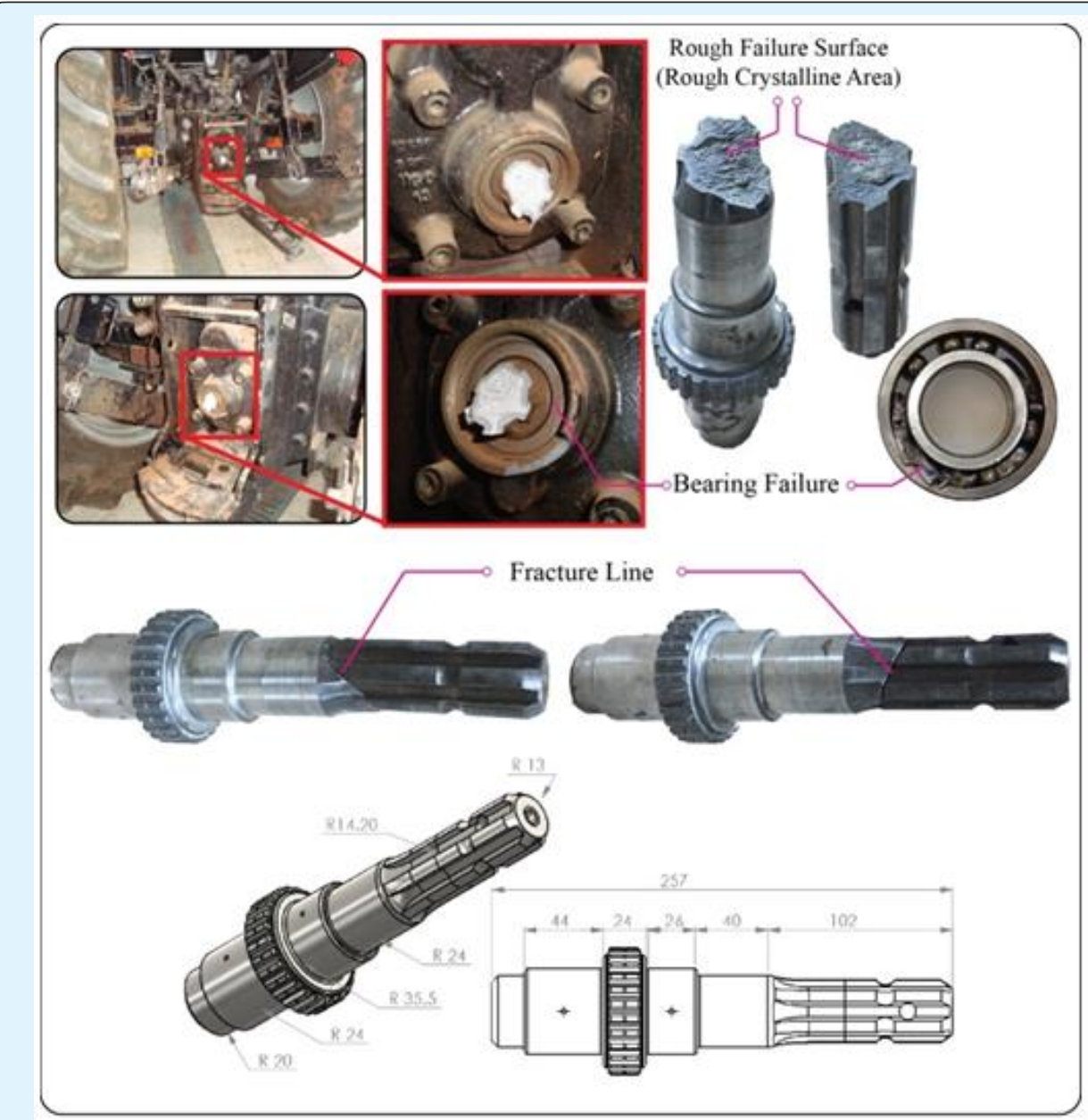

Figure 2: Failed PTO shaft. 


\section{Ergonomics International Journal}

\section{Finite Element Analysis}

The FEA was set up to simulate deformation behaviour and equivalent stress distribution on the PTO shaft under torsional loading. The most efficient transmission ( $90 \%)$ of net engine power (ASABE Standards, ASAE D497.7, 2015) for an agricultural tractor for a towed working implement requiring rotary power whether stationary or mobile is via the PTO shaft $[4,5]$. In consideration of this, the maximum torque of $830[\mathrm{~N} \cdot \mathrm{m}]$ (at $540\left[\mathrm{~min}^{-1}\right]$ ) applied to the PTO shaft was calculated through the tractor engine power [6]. The solid model of the shaft was reverse engineered in Solid Works parametric 3D CAD solid modelling software. ANSYS Workbench commercial FEA code was utilised for the simulation study. Linear static loading conditions and isotropic elastic material model assumptions were made in the FEA setup. The material considered in the PTO shaft was assumed as a medium-carbon steel denoted SAE 4140 (42CrMoS4), which is commonly used for induction hardened transmission components and drive shafts in the automotive industry [7-9]. The meshing functions of the ANSYS Workbench were used to create the Finite Element (FE) model (mesh structure) of the PTO Shaft [10]. The solving platform was a mobile workstation of Dell Precision M4800 Series (Intel Core $^{\mathrm{TM}}$ i7-4910MQ CPU @ 2.90GHz, NVIDIA-Quadro K2100M2GB, and Physical Memory-Total: 32 GB).

Determination of the appropriate element size is an important issue for FEA as the mesh structure is the critical factor in order to obtain an accurate result. Mesh density is a significant metric used to control accuracy of the FE model. Ideally, smaller elements can efficiently represent the solid model in a FEA, however, if the element size is too small, a larger amount of computer time may be required to solve the problem. A mesh sensitivity check can then be useful to decide upon the appropriate element size. To do this, a torsional loading scenario of the PTO was run with various element sizes and the effect of the different element sizes (from coarse to finer) on the deformation results was investigated. The mesh sensitivity study indicated that the minimum element size to sufficiently represent the torsional loading scenario of the PTO shaft with an acceptable computation time was $1[\mathrm{~mm}]$. A uniform element sizing strategy was accepted in creating the FE model (mesh structure) of the PTO shaft, and subsequently, the FE model was created. The boundary conditions, mesh sensitivity analysis, details of the final mesh structure and material properties used in the FEA set up are shown in Figure 3.

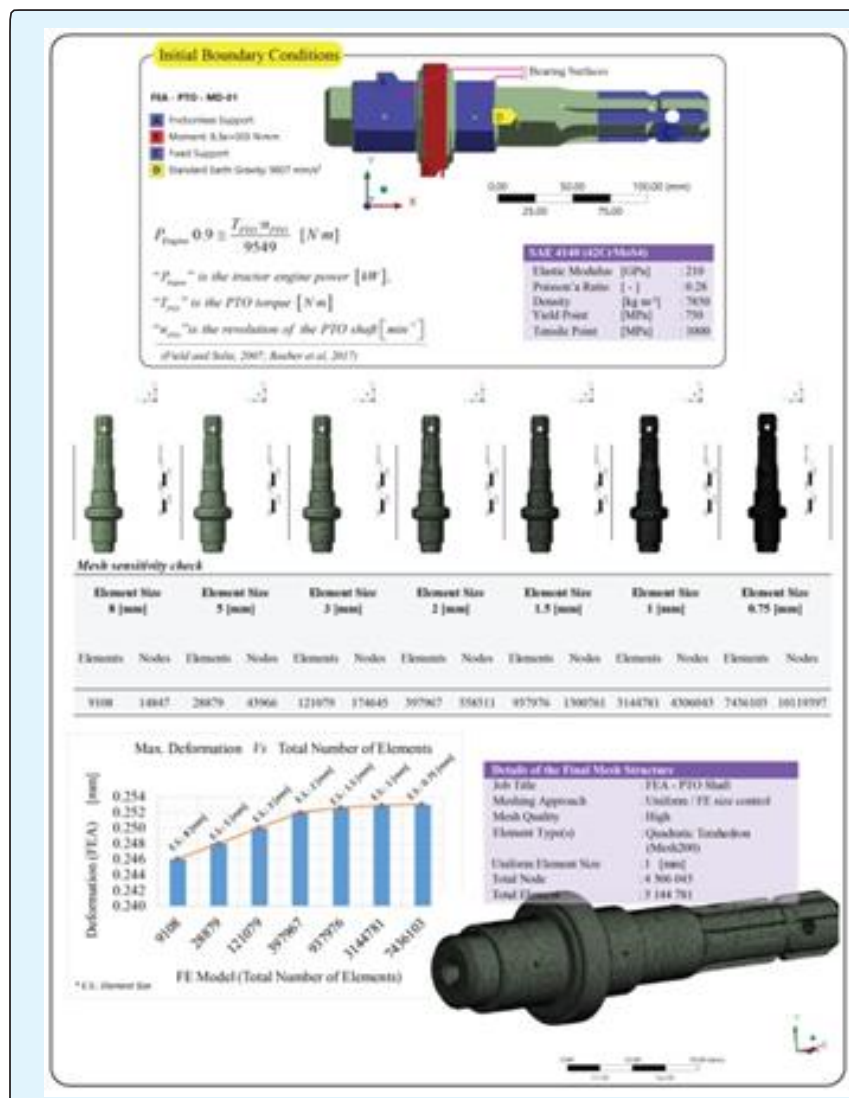

Figure 3: Initial FEA set up and mesh sensitivity check.

\section{Results and Discussions}

After all pre-processing procedures and definitions, the FEA simulation was run and the results were recorded. The numerical results extracted from the simulation showed that the maximum Von-Mises equivalent stress on the PTO shaft was $632.08[\mathrm{MPa}]$ and the maximum torsional deformation was 0.1855 [mm] (Figure 4). The equivalent stress magnitude obtained from the FEA did not exceed the material yield point $(750[\mathrm{MPa}])$. This indicated that the PTO shaft works in the elastic deformation zone under defined boundary conditions (maximum torsional loading).This magnitude was also lower than the material tensile point (1000 [MPa]) (the tensile point can be assumed to be the fracture failure limit in this static study) and this could not be the reason for the fracture failure. The shaft considered in this study was damaged with an angle close to $45\left[^{\circ}\right]$, which caused a rough, crystalline surface to form on the shaft, and the maximum torsional deformation was a low value $(0.1855[\mathrm{~mm}])$. 


\section{Ergonomics International Journal}

These were the indicators of brittle fracture under excessive torsion. Therefore, it would be correct to say that a dynamic loading case such as shock impact was responsible for this failure case (it was assumed that there were no other faults such as material inner structure, poor design or heat treatment).

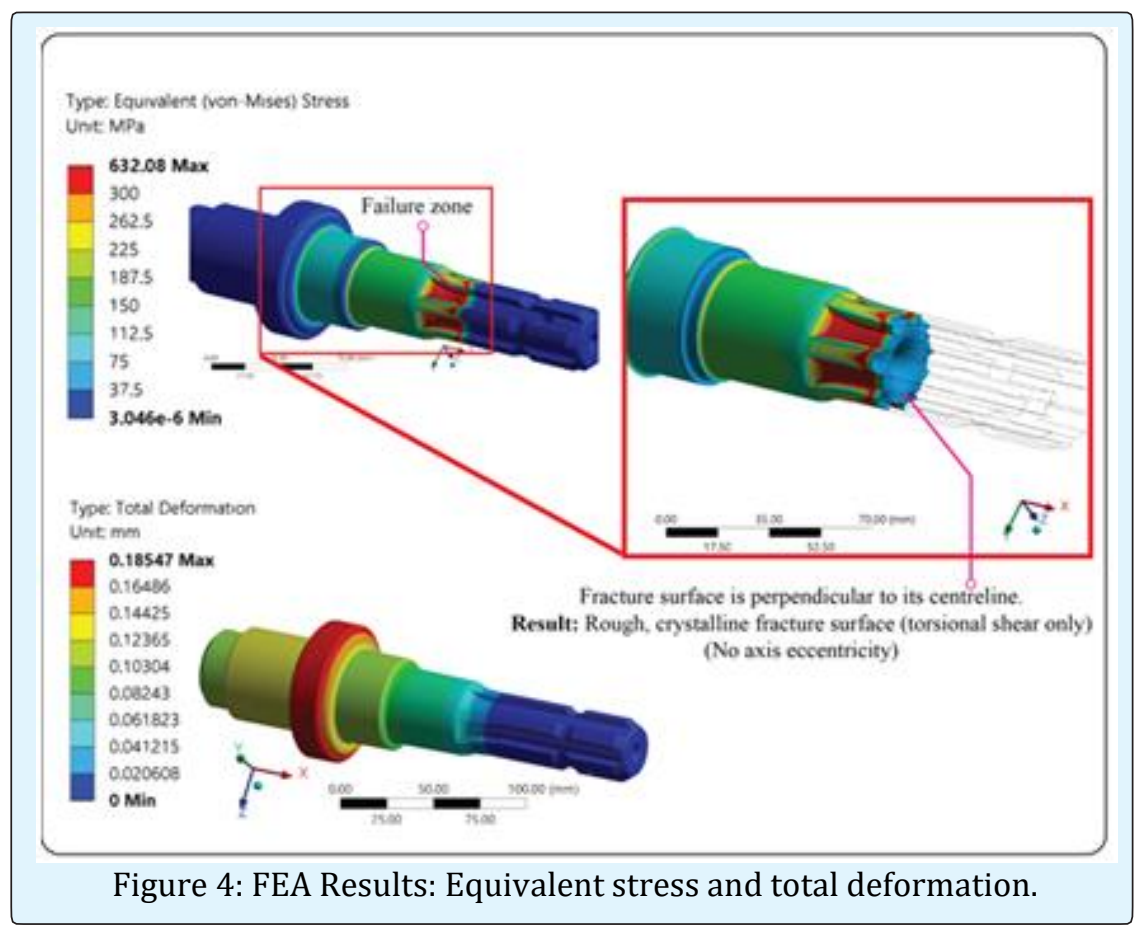

In fact, the result obtained from the FEA was reasonably well matched in order to determine the failure zone on the shaft, however, an exact visual fit could not be achieved in this FEA with the fracture type shown in Figure 2 This showed that although the failure zone on the shaft was successfully represented in the FEA results, a static linear torsional loading case only was not enough to represent the result of rapidly-applied twisting motion which is evaluated as the main reason for the failure of the PTO shaft shown in Figure 2.It means thata sudden loading case (dynamic shock loading) must be considered in such a failure analysis by means of FEA. Static and linear solving approach used in the FEA did not support part failure on the shaft model considered in the simulation, however, this may be provided by a deeper analysis that would be considered in any future work considering dynamic conditions with a nonlinear material model. This would help to obtain more accurate fracture surface results in a FEA [11].

\section{Conclusion}

Finite element analysis of a failed PTO shaft used in an agricultural tractor was discussed in detail in this paper. Initial visual observations indicated a brittle fracture failure on the failed PTO shaft. FEA results successfully determined the failure zone on the PTO shaft, however, exact fracture signs were not represented in the simulation. This led us to consider impact dynamic loading conditions such as a shock torsional loading case. In a similar context, although the static linear FEA approach revealed a deformation on the failure zone, it did not support proper part failure in the simulation. Torsional shock load results when a rapidly-applied twisting motion occurs; when an excessive amount of torque is delivered to an axle shaft. Shock load causes components to crack and separate from each other as a result of such failures. An operator speeds up the engine and rapidly releases the clutch("popping the clutch"), which causes an immediate force, or load, to the driveline. This was concluded as the main reason for failure of the shaft in this study.

Failures are frequently referred to as seriously affecting the production costs of agricultural operations. To avoid recurrence of the type of failure described in this study, it is recommended that operation of the tractor within its approved application and weight limits is an important failure prevention parameter. To design a PTO shaft with high service durability, mechanical characteristics such as fatigue strength, ultimate tensile strength, and fracture toughness are important properties 


\section{Ergonomics International Journal}

that should be considered in material selection at the design stage as well. This work contributes to further research into usage of numerical method based deformation simulation studies for the transmission/rotary elements used in agricultural tractors/machinery.

\section{Acknowledgements}

The abstract of this study was published and presented in XIX. World Congress of the International Commission of Agriculture and Biosystems Engineering (CIGR) (22-25 April, 2018, Antalya, Turkey). Additionally, the authors wish to acknowledge that this study is partly supported financially by The Scientific Research Projects Coordination Unit of Akdeniz University (Turkey) and Lancaster Product Development Unit (LPDU) in Lancaster University (United Kingdom).

\section{References}

1. Turkish Statistical Institute (2017) Agricultural Mechanisation statistics.

2. Triantafyllidis G, Zagkliveris D, Koliotsas P (2015) Metallurgical Analysis Explains the Failure Mechanism of a Farm Tractor's PTO + PTO's Drive Shaft, Assisting in the Evaluation of Economical Consequences of Similar Events, Journal of Failure Analysis and Prevention 15(2): 179-183.

3. Pardhi DG, Khamankar SD (2014) Stress Analysis of Spline Shaft Using Finite Element Method and its Experimental Verification by Photo Elasticity. Int J Mech Eng 3(4): 451-458.
4. ASABE Standards (2015) Agricultural Machinery Management Data. ASAE D497.7.

5. Roeber James BW, Pitla Santosh, Hoy Roger M, Luck Joe D, Kocher Michael F (2017) Tractor Power Take-off Torque Measurement and Data Acquisition System. Biological Systems Engineering: Papers and Publications 33(5): 679-686.

6. Field H, Solie J (2007) Introduction to Agricultural Engineering Technology: A Problem Solving Approach, Springer Science \& Business Media, pp: 391.

7. Starke P, Walther F, Eifler D (2009) New fatigue life calculation method for quenched and tempered steel SAE 4140. Mater Sci Eng A 523(1-2): 246-252.

8. Barsoum I, Khan F, Barsoum Z (2014) Analysis of the torsional strength of hardened splined shafts, Materials and Design 54: 130-136.

9. Kristoffersen H, Haglund S (2012) Influence of straightening on bending fatigue properties of induction hardened shafts in steel 42CrMo4 (SAE 4140). In: Proceedings of the 6th International Quenching and Control of Distortion Conference, Chicago.

10. Resolved Issues and Limitations (2017) ANSYS Documentation, Release notes: Meshing, Release 18.2. ANSYS Inc.

11. Arvin Meritor (2008) Manual TP-0445, Parts Failure Analysis, Supersedes Manual TP-87123 (Revised 0408). 